\title{
When your best friend turns on you; the impact of bad Propofol
}

\author{
Adam C. Adler
}

Received: 20 December 2014 / Accepted: 3 January 2015 / Published online: 17 February 2015

(C) Japanese Society of Anesthesiologists 2015

Keywords Propofol $\cdot$ Safety $\cdot$ Contamination

\section{To the Editor:}

Propofol has been one of the most revolutionary advances in the pharmaceutical arsenal of the anesthesiologist. The active ingredient 2,6-diisopropylphenol is a nonpyrogenic emulsion using soybean oil, glycerol and egg yolk phospholipid for suspension. While the lipid base is required for production it unfortunately supports microbial growth. To counteract this, sodium metabisulfite or EDTA has been added to inhibit extrinsic microorganism growth for up to $12 \mathrm{~h}$.

There have been many reports of propofol microbial contamination leading to fever, sepsis and even death [1]. The burden on surgical and intensive care unit-associated nosocomial infections may have been previously underestimated. However, these reports focus on patients in the intensive care unit following continuous propofol infusion and not on single dose vials that are used in anesthesia.

For multi-dose vials, if an extended period of time has elapsed between first and second administrations, providers must be wary of the possibility of bacterial propagation.

Electronic supplementary material The online version of this article (doi:10.1007/s00540-015-1976-1) contains supplementary material, which is available to authorized users.

A. C. Adler $(\bowtie)$

Department of Anesthesiology and Critical Care Medicine, The Children's Hospital of Philadelphia, Philadelphia, USA

e-mail: adlera@email.chop.edu

A. C. Adler

Perelman School of Medicine of the University of Pennsylvania, Philadelphia, USA
Supplementary Image 1 demonstrates the appearance of significant discoloration of a propofol vial at the end of a long case. Despite knowledge of propofol as a potential vehicle for bacterial transfer to our patients, we continue to receive reports of propofol-induced sepsis. It is difficult to identify the anesthetic as the cause of contamination. It is incumbent upon the anesthesiology care provider to maintain strict and vigilant practices when using propofol. Providers should be reminded of the dangers that propofol contamination poses to patients and continue employing preventative measures.

Recommendations for reduction of extrinsic contamination of propofol include [2]:

- Use of strict aseptic technique.

- Scrubbing of the vial's rubber stopper with $70 \%$ isopropyl alcohol prior to each withdrawal.

- Use of a new, sterile syringe for each administration.

- Flushing of IV tubing at the end of an anesthetic or changing IV tubing every $12 \mathrm{~h}$ during long cases.

- Drawing up propofol immediately prior to administration and capping all remaining syringes.

- Discarding propofol after $12 \mathrm{~h}$ or at any signs of contamination.

Conflict of interest The authors have no relevant conflicts of interest to report.

\section{References}

1. Muller AE, Huisman I, Roos PJ, Rietveld AP, Klein J, Harbers JB, Dorresteijn JJ, van Steenbergen JE, Vos MC. Outbreak of severe sepsis due to contaminated propofol: lessons to learn. J Hosp Infect. 2010;76(3):225-30.

2. Trépanier CA, Lessard MR. Propofol and the risk of transmission of infection. Can J Anaesth. 2003;50(6):533-7. 